\section{- OPEN ACCESS}

\title{
Awakening with amantadine from a persistent vegetative state after subarachnoid haemorrhage
}

\author{
Sophie Mirabell Lehnerer, ${ }^{1}$ Franziska Scheibe, ${ }_{1}^{1}$ Ralph Buchert, ${ }_{1}^{2}$ Stefan Kliesch, ${ }^{3}$ \\ Andreas Meisel ${ }^{1}$
}

'Department of Experimenta Neurology, NeuroCure Clinical Research Center, and Department of Neurology, Charite'-University Medicine Berlin, Berlin, Germany ${ }^{2}$ Department of Nuclear Medicine, Charite'-University Medicine Berlin, Berlin, Germany ${ }^{3}$ Department of Neuroradiology, Charite'-University Medicine Berlin, Berlin, Germany

Correspondence to Dr Sophie Mirabell Lehnerer, sophie.lehnerer@charite.de

Accepted 11 June 2017

\footnotetext{
To cite: Lehnerer $S M$, Scheibe F, Buchert R, et al. BMJ Case Rep Published Online First: [please include Day Month Year]. doi:10.1136/bcr-2017220305
}

\begin{abstract}
SUMMARY
We report the case of a 36-year-old woman with a subarachnoid haemorrhage ( $\mathrm{SAH}$ ) caused by a rupture of a right-sided middle cerebral artery aneurysm and subsequent malignant infarction of the right hemisphere leading to a persistent vegetative state and severe spastic tetraparesis with recurrent myocloni. Nine months after disease onset, the patient was transferred to our department for diagnostic and therapeutic re-evaluation. The poor clinical condition could not be explained by the brain lesion caused by the SAH or infarction. Moreover, glucose metabolism was normal in brain regions not affected by SAH and infarction as shown by positron emission tomography with ${ }^{18} \mathrm{~F}$-fluorodeoxyglucose. We terminated baclofen and reduced antiepileptics known to impair vigilance and cognitive functions. However, only after starting amantadine treatment we observed a stunning awakening of the patient fully orientated within days. Our findings warrant trials to investigate amantadine in the treatment of unresponsive wakefulness syndromes due to acute central nervous system diseases.
\end{abstract}

\section{BACKGROUND}

Different causes of brain damage (eg, global ischemia, hypoxia, infarction, bleeding, trauma, or infection) can result in unresponsive wakefulness syndromes. Evaluation of cognitive functions in these patients is difficult and requires a precise clinical examination supported by additional investigations like electrophysiological studies, brain MRI and positron emission tomography (PET). Contradictive findings of clinical syndrome and diagnostic test results should direct physicians to therapeutic consequences like reduction, discontinuation or substitution of drugs affecting brain function, such as sedatives or antiepileptics. Moreover, the addition of neurostimulant drugs should be considered.

\section{CASE PRESENTATION}

A 36-year-old woman suffered from a subarachnoid haemorrhage (SAH) after rupture of a right-sided middle cerebral artery aneurysm. Due to a secondary vasospastic malignant infarction of the right middle cerebral artery territory, a haemicraniectomy was performed. A subsequent posthaemorrhagic cerebrospinal fluid circulatory dysfunction required implantation of a ventriculoperitoneal shunt. After initial coma, the patient was affected by a left-sided hemiparesis, but was able to communicate on a basic level and could execute simple actions like drinking from a feeding cup (for overview of clinical course see figure 1). During rehabilitation the condition of the patient deteriorated over several weeks. Finally, she became unresponsive to external stimuli and showed vegetative dysfunction such as vomiting, extreme sweating, tachypnoea and tachycardia. After correcting a suspected overdrainage of the ventriculoperitoneal shunt and cranioplastic skull reconstruction 5 months after the initial bleeding, the patient did not return to her best neurological status after brain haemorrhage. In particular she presented an unresponsive wakefulness syndrome. In the further course she was weaned from respirator breathing spontaneously through a tracheal cannula. Several complications like infected decubiti, upper limb phlegmon, pneumothorax, anaemia, anovesical fistula and multiple infections including pneumonia, urinary tract infection and catheter-associated sepsis resulted in a prolonged stay on the intensive care unit (ICU). Enhanced by stressful stimuli, she additionally presented a rhythmic myoclonus of the right upper and lower limbs and mandible muscles including the tongue. A tremor was considered as a differential diagnosis, but did not respond to pramipexole. Three antiepileptic drugs (levetiracetam $4 \mathrm{~g} /$ day, valproic acid $300 \mathrm{mg} /$ day, clonazepam $1.5 \mathrm{mg} /$ day) were used without effect on the myoclonus. Noteworthy, the patient showed no seizures and no epileptic activity had been demonstrated in several electroencephalographies (EEGs). The prognosis of the patient was considered to be poor with no options for further treatments beyond home care nursing or an end-of-life decision by the maximum care hospital. Initiated by her family, the patient was transferred to our university hospital for a second opinion including re-evaluation of prognosis and treatment options 9 months after disease onset.

\section{INVESTIGATIONS}

Considering the subacute progression into the persistent vegetative state as well as the severe tetraparesis, which was at least in part not explained by the initial brain damage, we discussed possible causes for the late-onset deterioration like another ischaemic or haemorrhagic infarction, over or underdrainage of the ventriculoperitoneal shunt, septic encephalopathy due to multiple bacterial infections, side effects of medication or central nervous system (CNS) infection due 


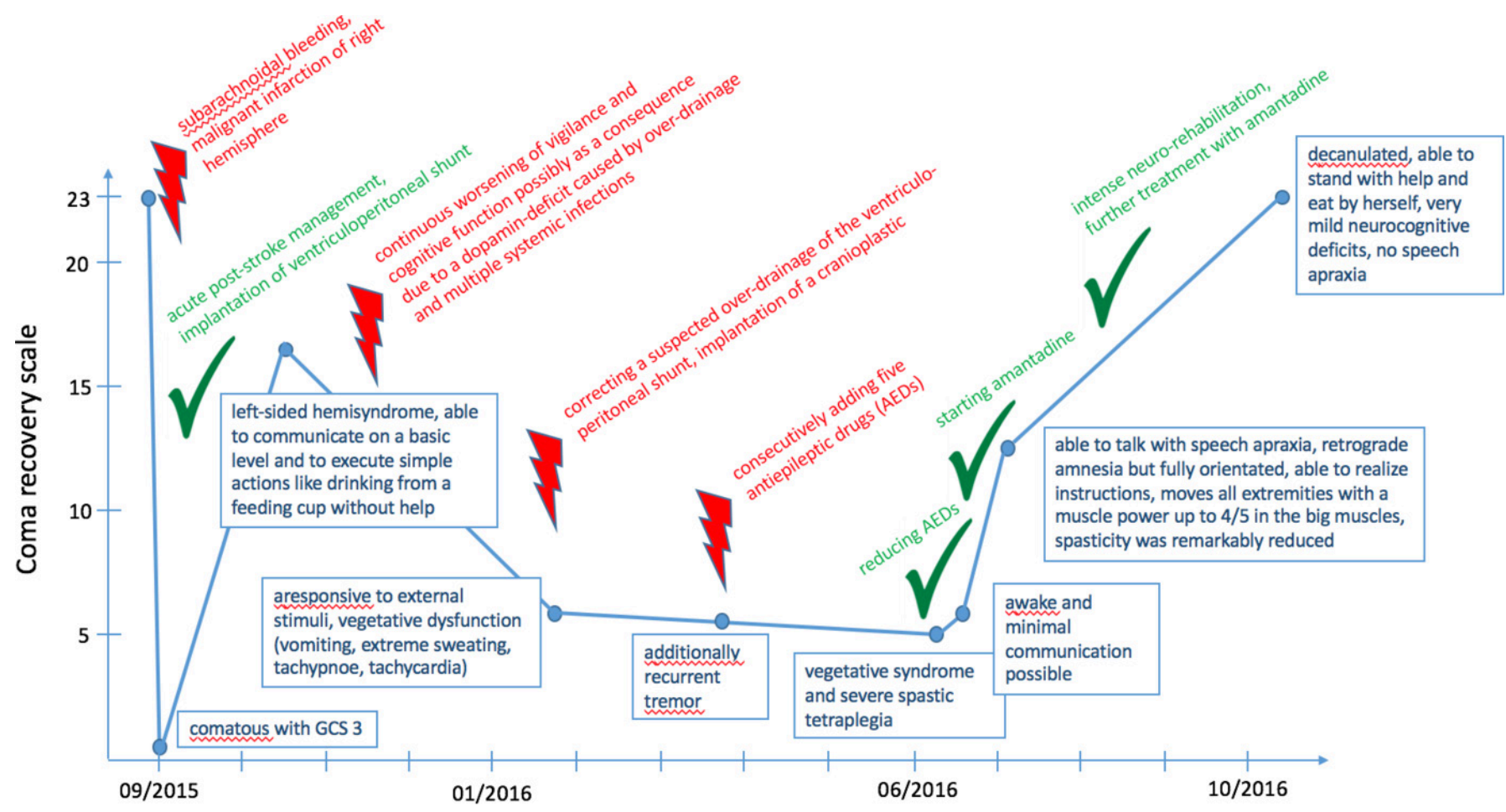

Figure 1 Schematic overview of the clinical course from the initial event (subarachnoid haemorrhage) to the improvement of vigilance, cognitive function and motor symptoms 13 months later. The graph shows the CRS-R. The blue boxes provide additional information about the clinical condition. Events worsening the status of our patient are marked with red arrows, interventions with a positive effect are highlighted by green checks. Some of the CRS-R scores have not been mentioned explicitly in the reports of former hospitals but were extrapolated from detailed medical reports. AED, antiepileptic drug; CRS-R, revised coma recovery scale; GCS, Glasgow Coma Scale.

to shunt implantation. First we performed a cranial CT that showed no signs of over or underdrainage of the ventriculoperitoneal shunt. MRI of the brain exhibited a superficial siderosis and laminar necrosis in the territory of the former right middle cerebral artery infarction with temporal, frontal and opercular atrophies, and consequently widened ventricle of the right hemisphere (figure 2). Although the patient presented clinically with a severe proportional spastic tetraparesis, brain and spinal MRI exhibited structural abnormalities neither in the left hemisphere nor in the brainstem and myelon. Consistent with the MRI findings, PET with the glucose analogue ${ }^{18} \mathrm{~F}$-fluorodeoxyglucose (FDG) revealed normal glucose metabolism in the brain except

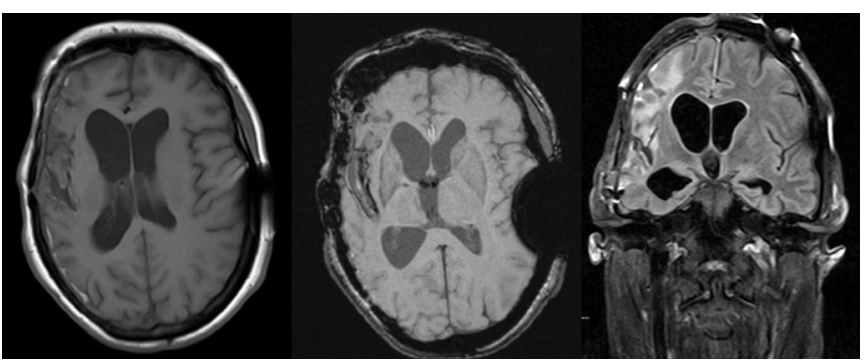

Figure 2 Axial T1SE (left), susceptibility-weighted (middle) and coronal fluid attenuated inversion recovery (right) MR images obtained during re-evaluation at our institution reveal defectassociated volume loss and signs of laminar necrosis in the area of the chronic middle cerebral artery infarction. SWI shows residual superficial siderosis due to the subarachnoid haemorrhage. Artefact due to valve of the ventriculoperitoneal shunt system pronounced in SWI. SWI, susceptibility-weighted imaging. in the infarction area (figure 3). Cerebral glucose metabolism as measured by FDG-PET serves as a surrogate marker for synaptic signalling in the brain sensitive to focal structural damage and dysfunction and to interruption of complex brain networks. Considering these findings we expected rather a focal neurological deficit but not a global brain dysfunction.

Neuroinflammation as underlying cause of the condition is not excluded but rather unlikely due to normal findings in the examination of the cerebrospinal fluid including cell counts, lactate, glucose and protein levels as well as negative antibodies for autoimmune disorders of the nervous system. Investigated under analgosedation, about $50 \%$ of movement restriction was attributed to spasticity and the other $50 \%$ to primarily distal located fixed contractions.

\section{TREATMENT}

Since oral baclofen $(30 \mathrm{mg} /$ day) given over several months had no significant effect on spasticity, we tapered it off. In addition, we tested responsiveness to baclofen by increasing dosages administered intrathecally. Even repeated dosages of $300 \mu \mathrm{g}$ baclofen had insignificant effects on the spasticity. Since we suspected among other causes an antiepileptic drug-induced encephalopathy, we reduced and finally stopped levetiracetam, valproic acid and clonazepam. Although cessation of the antiepileptic drugs did not increase the myoclonus, it did not improve cognitive functions. Due to a single self-limiting generalised epileptic seizure associated with a prior intrathecal baclofen injection, we treated the patient with lamotrigine. In repeated EEGs no epileptiform activity was noted.

We supposed the myoclonus in our patient was caused by a dopamine deficiency. Amantadine is known to increase indirectly 

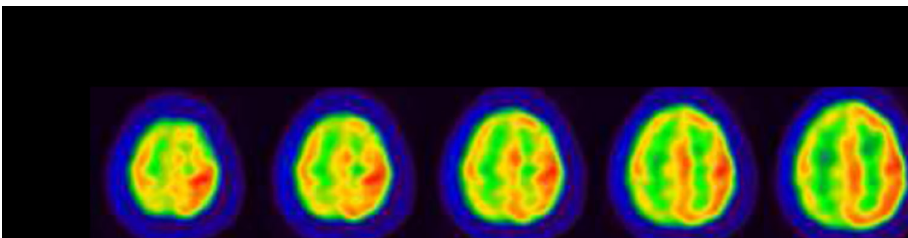

$+62$

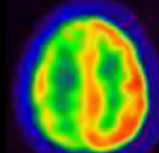

$+42$

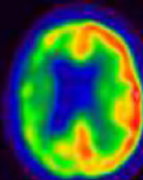

$+22$

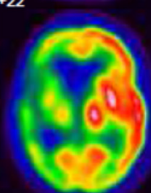

$+2$

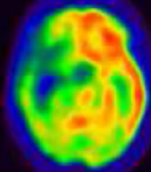

$-18$

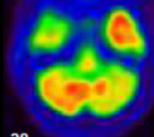

$+58$

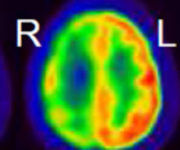

$+38$

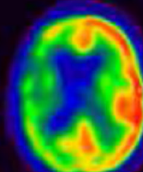

$+18$

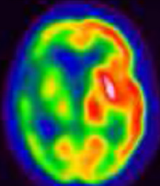

$-2$

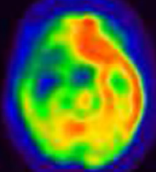

$-22$

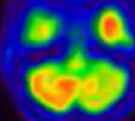

$+54$

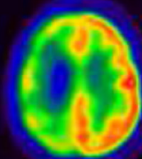

$+34$

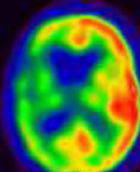

$+14$
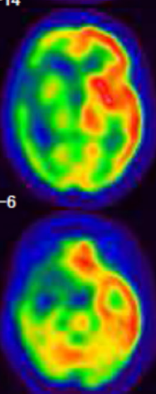

$-26$

$+50$

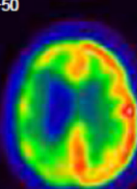

$+30$

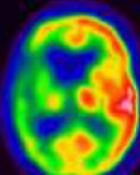

$+10$
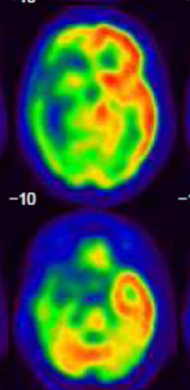

$-30$

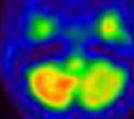

$-46$
$+46$

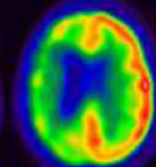

$+26$

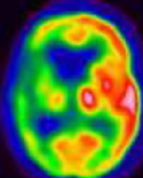

$+6$

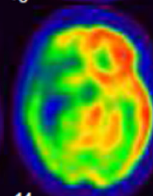

$-14$

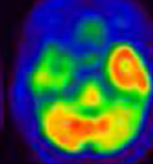

$-34$

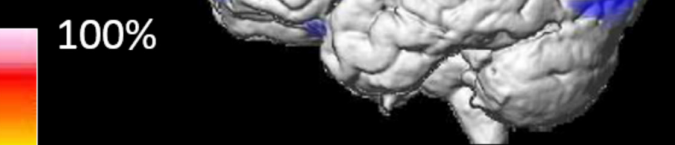

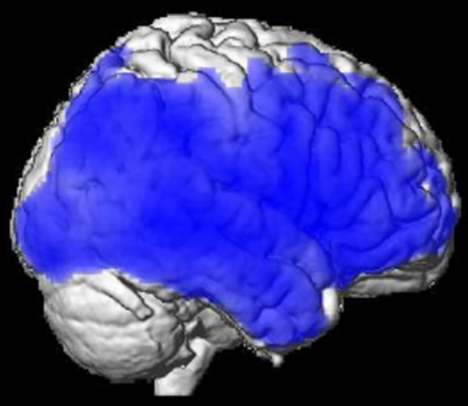

$P<0.001$

Figure 3 PET of the brain with the glucose analogue FDG 9 months after disease onset. Transversal slices (left) show a large frontotemporal defect in the right hemisphere consistent with the area of the cerebral infarction (figure 2). Glucose metabolism in the left hemisphere appears normal by visual inspection. This was confirmed by voxelwise statistical testing of the patient's FDG-PET against a database of healthy subjects (right). Brain areas with significantly reduced metabolism are indicated in blue in the render display of the statistical parametric map. FDG, ${ }^{18} \mathrm{~F}-$ fluorodeoxyglucose; PET, positron emission tomography.

dopamine via antagonistic effects at the N-methyl-D-aspartate (NMDA) receptor by increasing the release and blocking the reuptake of dopamine. Since amantadine might also enhance arousal from coma due to dopamine effects, ${ }^{1}$ we initiated a therapy with amantadine starting with $100 \mathrm{mg} /$ day intravenously, increasing the dose $100 \mathrm{mg}$ per day, and switched to an enteral drug administration after reaching the maximum dose of $300 \mathrm{mg}$ /day given once in the morning.

\section{OUTCOME AND FOLLOW-UP}

Under this treatment the patient improved within 16 days from initially 5 points of the revised coma recovery scale to the maximum score of 23 points. With a speaking valve she was able to talk, although a speech apraxia was seen (see online supplementary video 1). Our patient had a retrograde amnesia but she was subsequently fully orientated and was able to communicate adequately in two to six-word sentences. Being able to realise instructions she could move all extremities with a muscle power up to $4 / 5$ in the big muscles. Upon these active movements the spasticity was remarkably reduced. The myoclonus of the right extremities and the tongue stopped apart from rare situations when the patient was agitated. For further improvement of motor and cognitive functions we transferred the patient to a specialised neurorehabilitation centre. Visiting the patient 3 months later, we saw a fully oriented patient in much improved general condition. Apart from a retrograde amnesia regarding her stay on the ICUs, she presented only very mild cognitive deficits (see online supplementary video 2). By that time she was decanulated, eating autonomously precut food (see online supplementary video 3 ) and was able to stand with help. Walking was still difficult due to fixed contractions (see online supplementary video 4 ).

\section{DISCUSSION}

Here we describe a patient with an unexplained unresponsive wakefulness syndrome accompanied by a tetraparesis due to a SAH complicated by a malignant infarction in the right middle cerebral artery territory and severe systemic bacterial infections in the chronic course. The patient improved significantly under amantadine treatment to remaining moderate motor deficits and minor cognitive dysfunction. 
Amantadine was introduced in the 1960s for the treatment of the influenza A virus. Although it lost its importance in the antiviral treatment, amantadine is still frequently used in the treatment of Parkinson's disease. ${ }^{2}$ Amantadine's mechanism of action is still not completely understood, but it is thought to act as a non-competitive NMDA receptor antagonist increasing dopamine synthesis and dopamine release in the striatum. Sufficient synaptic dopamine levels are necessary for a number of physiological functions including movement control, emotion, motivation and cognitive processing. ${ }^{3}$ Amantadine has successfully been evaluated for neurostimulation in patients with traumatic brain injury (TBI) in a multicentre trial. ${ }^{4}$ Mechanistically, severe brain trauma leads to diffuse axonal injury which results among other in a reduced dopamine turnover. ${ }^{5}$ There are no trials investigating amantadine for the treatment of cognitive dysfunctions following other causes of cerebral damage such as stroke, intracranial bleeding or hypoxia.

Most clinical studies report the use of amantadine within the first weeks after the initial event. In our patient, we observed dramatic improvement on amantadine treatment 9 months after the initial brain damage. The improvement encompassed quantitative and qualitative aspects of consciousness and the severe tetraparesis and myoclonus.

We suppose that the myoclonus of the right limbs and mandible muscles including the tongue resulted from dopamine deficiency that was effectively treated by amantadine. Some patients with TBI present parkinsonian symptoms associated with mild brain dysfunction. ${ }^{6}$ There might have been similar midbrain dysfunction in our patient caused by overdrainage of the ventriculoperitoneal shunt, which is however unlikely since we have not observed a definite overdrainage. A further possible rare cause of myoclonus in our patient is a sepsis-associated encephalopathy.

Our report of awakening on amantadine treatment in a patient suffering from a chronic course of a severe CNS lesion emphasises the importance of a careful neuroprognostic (re) evaluation considering the medical history, current clinical findings as well as state-of-the art neuroimaging. Cerebral FDG-PET could be used to complement bedside examinations and predict long-term recovery of patients with unresponsive wakefulness syndrome. ${ }^{7}$ In our patient, the PET showed hypometabolism only in the area of the malignant infarction of the right medial cerebral artery, which could not explain the persistent vegetative state and the spastic tetraparesis. Even in the chronic course after severe brain damage discrepancies between the clinical condition and paraclinical findings should initiate a re-evaluation of the medication, in particular one should consider termination of drugs known to affect neuronal function, such as antiepileptic or neuroleptic agents. Moreover, treatment should consider the use of neurostimulants such as amantadine. Several further approaches to stimulate arousal in patients with unresponsive wakefulness syndromes or coma have been described including pharmacological therapy with ketamine, bromocriptine, zolpidem or methylphenidate as well as non-pharmacological interventions such as transcranial magnetic stimulation or deep brain stimulation. ${ }^{8}$ The personalised approach of careful re-evaluation and trying different therapy options is often time and personal intensive requiring a highly specialised team of professionals trained in neurological intensive care and in neuroprognostics.

\section{Learning points}

- Discrepancies between the clinical condition and paraclinical findings of patients with disorders of consciousness should initiate a re-evaluation, even in the chronic course after severe brain damage.

- Cerebral ${ }^{18} \mathrm{~F}$-fluorodeoxyglucose-positron emission tomography could be used to complement bedside examinations and to predict long-term recovery of patients with unresponsive wakefulness syndrome.

- For drugs known to affect neuronal function, such as antiepileptics or neuroleptics, a dose reduction or even termination should be considered under careful monitoring.

- Neurostimulants such as amantadine should be considered in cases of minimal conscious state or even unresponsive wakefulness syndrome.

Contributors SML: design and conceptualisation of the case report, involved in clinical care, acquisition of data, analysis and interpretation of data, drafting of manuscript. FS: involved in clinical care, acquisition and interpretation of data revision of manuscript for intellectual content. RB: acquisition, description and interpretation of PET data, revision of manuscript for intellectual content. SK: acquisition, description and interpretation of MRI data, revision of manuscript for intellectual content. A. Meisel: involved in conceptualisation of the case report, interpretation of data, revision of manuscript for intellectual content.

Competing interests None declared.

Patient consent Obtained.

Provenance and peer review Not commissioned; externally peer reviewed.

Open Access This is an Open Access article distributed in accordance with the Creative Commons Attribution Non Commercial (CC BY-NC 4.0) license, which permits others to distribute, remix, adapt, build upon this work non-commercially, and license their derivative works on different terms, provided the original work is properly cited and the use is non-commercial. See: http://creativecommons.org/ licenses/by-nc/4.0/

(C) BMJ Publishing Group Ltd (unless otherwise stated in the text of the article) . All rights reserved. No commercial use is permitted unless otherwise expressly granted.

\section{REFERENCES}

1 Sawyer E, Mauro LS, Ohlinger MJ. Amantadine enhancement of arousal and cognition after traumatic brain injury. Ann Pharmacother 2008;42:247-52.

2 Connolly BS, Lang AE. Pharmacological treatment of Parkinson disease: a review. JAMA 2014;311:1670-83.

3 Bales JW, Wagner AK, Kline AE, et al. Persistent cognitive dysfunction after traumatic brain injury: a dopamine hypothesis. Neurosci Biobehav Rev 2009;33:981-1003.

4 Giacino JT, Whyte J, Bagiella E, et al. Placebo-controlled trial of amantadine for severe traumatic brain injury. N Engl J Med 2012;366:819-26.

5 Meythaler JM, Brunner RC, Johnson A, et al. Amantadine to Improve Neurorecovery in traumatic brain Injury-Associated Diffuse Axonal Injury. J Head Trauma Rehabil 2002;17:300-13.

6 Formisano R, Zasler ND. Posttraumatic parkinsonism. J Head Trauma Rehabil 2014;29:387-90.

7 Stender J, Gosseries O, Bruno MA, et al. Diagnostic precision of PET imaging and Functional MRI in disorders of consciousness: a Clinical validation study. Lancet 2014;384:514-22.

8 Cossu G. Therapeutic options to enhance Coma arousal after traumatic brain injury: state of the art of current treatments to improve Coma recovery. $\mathrm{Br} J$ Neurosurg 2014;28:187-98. 
Copyright 2017 BMJ Publishing Group. All rights reserved. For permission to reuse any of this content visit http://group.bmj.com/group/rights-licensing/permissions.

BMJ Case Report Fellows may re-use this article for personal use and teaching without any further permission.

Become a Fellow of BMJ Case Reports today and you can:

- Submit as many cases as you like

- Enjoy fast sympathetic peer review and rapid publication of accepted articles

- Access all the published articles

- Re-use any of the published material for personal use and teaching without further permission

For information on Institutional Fellowships contact consortiasales@bmjgroup.com

Visit casereports.bmj.com for more articles like this and to become a Fellow 